\title{
Dynamic fluctuations in a Short-Range Spin Glass model
}

\author{
Paola Ranieri
}

April 20, 2017

\author{
Dipartimento di Fisica, Università di Roma La Sapienza, \\ P. Aldo Moro 2, 00185 Roma, Italy
}

\begin{abstract}
We study the dynamic fluctuations of the soft-spin version of the Edwards-Anderson model in the critical region for $T \rightarrow T_{c}^{+}$. First we solve the infinite-range limit of the model using the random matrix method. We define the static and dynamic 2-point and 4-point correlation functions at the order $O(1 / N)$ and we verify that the static limit obtained from the dynamic expressions is correct. In a second part we use the functional integral formalism to define an effective short-range Lagrangian $L$ for the fields $\delta Q_{i}^{\alpha \beta}\left(t_{1}, t_{2}\right)$ up to the cubic order in the series expansion around the dynamic Mean-Field value $\overline{Q^{\alpha \beta}}\left(t_{1}, t_{2}\right)$. We find the more general expression for the time depending non-local fluctuations, the propagators $\left[\left\langle\delta Q_{i}^{\alpha \beta}\left(t_{1}, t_{2}\right) \delta Q_{j}^{\alpha \beta}\left(t_{3}, t_{4}\right)\right\rangle_{\xi}\right]_{J}$, in the quadratic approximation. Finally we compare the long-range limit of the correlations, derived in this formalism, with the correlations of the infiniterange model studied with the previous approach (random matrices).
\end{abstract}




\section{Introduction}

The static and dynamic Mean Field (MF) theory of Spin Glasses (SG) systems for $T \geq T_{c}$ is well defined and understood. This theory has been studied through different approaches all consistent among themselves. Many important results concerning the equilibrium static properties of SG have been derived using the replica method [1]. Sompolinsky and Zippelius [3], [4], 6] studied a soft spin version of the Edwards-Anderson model with the dynamic formalism, avoiding the replica trick. They defined a Langevin dynamics on the system and analysed the infinite range limit where the MF solution is exact. The static limit derived from the dynamic expressions is in agreement with the static prevision obtained with replica. Moreover, dynamic characteristics of the model have been well defined.

Unfortunately the behaviour of short-range SG system is not clear yet. There are different analytic works and simulations about the Ising and Heisemberg SG in finite dimensions, [8], 9], [10], [11, that do not agree with each other.

On the other hand, in order to study the corrections to the MF behaviour of the Green functions one can use the Renormalization Group theory and the $\epsilon$-expansion. Chen, Lubensky [16], [17] and Green [18] studied a static Ising model in $d=6-\epsilon$ dimensions (6 is the upper critical dimension) with the Replica method and they found the corrections to the second order in $\epsilon$ for the critical exponents.

In this work, we want investigate the behaviour of the dynamic fluctuations of the short range SG in the critical region for $T \rightarrow T^{+}$. We study the softspin version of the Edwards-Anderson model that evolves through Langevin dynamics. We adopt the same procedure that Sompolinsky and Zippelius

used in [5], and we manage to explicit write the time-dependent propagators 
$\left[\left\langle\delta Q_{i}^{\alpha \beta}\left(t_{1}, t_{2}\right) \delta Q_{j}^{\alpha \beta}\left(t_{3}, t_{4}\right)\right\rangle_{\xi}\right]_{J}$ for any value of $t_{1}, t_{2}, t_{3}, t_{4}$ in the critical region, while in [5] they were defined only in the limit of two complete time separation. They are the elementary building blocks in a renormalization group calculation expanded in $\epsilon=6-d$ and can be used for future studies of the dynamic effects of higher-order terms (the cubic interactions). For evaluating these propagator we define an effective Lagrangian of the fields $\delta Q_{i}^{\alpha \beta}\left(t, t^{\prime}\right)$ (which represent the fluctuations around the MF order parameter value $\left.\overline{Q_{i}^{\alpha \beta}}\left(t, t^{\prime}\right)\right)$ through the functional integral formulation of the dynamics. In order to have a comparative term order by order in perturbation theory, we solve the infiniterange limit and the $\mathrm{O}(1 / \mathrm{N})$ corrections of this dynamic model, by using the distribution of the eigenvalues of the random interaction matrix. We verify that the expressions derived with the two different and independent methods are consistent each other.

The aim of this work is to pursue the study of the quadratic fluctuations of the soft-spin model in the general case, without having recourse to the Glauber model (hard-spin limit) [7]. We define the quadratic fluctuations as a perturbative series in $g$ (the coupling constant of the fourth order term of the soft-spin Lagrangian) which we succeed in resuming and therefore in obtaining a $g$ independent expression that could be directly used for further diagrammatic expansions of the theory. The results are qualitatively different from those obtained by Zippelius [7] and this may be related to the approximations done in going to the hard case.

This paper is organized as follows: in section 2 we define the theory; in section 3 we find the form of the quadratic corrections to the MF limit (order $O(1 / N)$, where $N$ is the sites number) using the diagonalization of the interaction random matrix; finally in section 4 we find the general propagator for an effective short range Lagrangian of the field $\delta Q^{\alpha \beta}\left(t, t^{\prime}\right)$ (fluctuations around MF limit); in the conclusion we present the possible development of this work. 


\section{Definition of the model}

Let us consider the soft spin version of the Edwards-Anderson model given by the Hamiltonian

$$
\beta \mathcal{H}=-\beta \sum_{\langle i j\rangle} \beta J_{i j} s_{i} s_{j}+\frac{1}{2} r_{0} \sum_{i} s_{i}^{2}+\frac{1}{4 !} g \sum_{i} s_{i}^{4},
$$

where the sum $\langle i j\rangle$ is over $z$ nearest-neighbor sites and the couplings $J_{i j}$ are quenched random variables with the following distribution:

$$
P\left(J_{i j}\right)=\frac{1}{(2 \pi z)^{1 / 2}} \exp \left(-z J_{i j}^{2} / 2\right) .
$$

A purely relaxational dynamics can be described by the Langevin equation:

$$
\Gamma_{0}^{-1} \frac{\partial s_{i}(t)}{\partial t}=-\frac{\partial(\beta \mathcal{H})}{\partial s_{i}(t)}+\xi_{i}(t)
$$

$\xi_{i}(t)$ is a Gaussian and white noise with zero mean and variance

$$
\left\langle\xi_{i}(t) \xi_{j}\left(t^{\prime}\right)\right\rangle=\frac{2}{\Gamma_{0}} \delta_{i j} \delta\left(t-t^{\prime}\right),
$$

which ensures that locally the fluctuation-dissipation theorem holds.

In the MF theory the physical quantities of interest are the average local (at the same point) correlation function

$$
C\left(t-t^{\prime}\right)=\left[\left\langle s_{i}(t) s_{i}\left(t^{\prime}\right)\right\rangle_{\xi}\right]_{J}
$$

and the average local response function

$$
G\left(t-t^{\prime}\right)=\frac{\Gamma_{0}}{2}\left[\left\langle s_{i}(t) \xi_{i}\left(t^{\prime}\right)\right\rangle_{\xi}\right]_{J}
$$

where the angular brackets refer to averages over the noise $\xi_{i}$ and square brackets over quenched disorder $J_{i j}$. 
Beyond the MF approximation we evaluate the non local fluctuations that are non vanishing

$$
\begin{aligned}
& G^{\alpha \beta \gamma \delta}\left(i-j ; t_{1}, t_{2}, t_{3}, t_{4}\right)= \\
& \quad\left[\left\langle\phi_{i}^{\alpha}\left(t_{1}\right) \phi_{i}^{\beta}\left(t_{2}\right) \phi_{j}^{\gamma}\left(t_{3}\right) \phi_{j}^{\delta}\left(t_{4}\right)\right\rangle_{\xi}\right]_{J}-\left[\left\langle\phi_{i}^{\alpha}\left(t_{1}\right) \phi_{i}^{\beta}\left(t_{2}\right)\right\rangle_{\xi}\right]_{J}\left[\left\langle\phi_{j}^{\gamma}\left(t_{3}\right) \phi_{j}^{\delta}\left(t_{4}\right)\right\rangle_{\xi}\right]_{J},(6)
\end{aligned}
$$

where $\alpha, \beta, \gamma, \delta$ can take the values 1 or 2 being $\phi_{i}^{1}=\xi_{i}$ and $\phi_{i}^{2}=s_{i}$. We shall see that these quantities represent the propagators of our theory. We will focus on the properties of the small frequency and small momentum of the propagators that have a critical slowing down near $T_{c}$.

In this formalism the time-depending spin-glass susceptibility is

$\chi_{S G}\left(i-j ; t_{1}-t_{3}, t_{4}-t_{2}\right)=\frac{1}{N}\left[\left\langle s_{i}\left(t_{1}\right) \xi_{j}\left(t_{3}\right)\right\rangle_{\xi}\left\langle s_{j}\left(t_{4}\right) \xi_{i}\left(t_{2}\right)\right\rangle_{\xi}\right]_{J}=G_{0}^{1221}\left(i-j ; t_{1}-t_{3}, t_{4}-t_{2}\right)$,

where the $G_{0}^{\alpha \beta \gamma \delta}$ functions represent the fluctuations in the limit $\left|t_{1}-t_{2}\right| \rightarrow \infty$ and $\left|t_{3}-t_{4}\right| \rightarrow \infty$.

\section{$3 \quad$ Mean Field limit and quadratic fluctuations by diagonalization of the interaction} matrix

The theory defined with the Hamiltonian (1) where the $J_{i j}$ are quenched random interactions can be solved by using the general properties of random matrices. It is convenient to apply this approach to an infinite range model $(z=N$ and $N \rightarrow \infty)$, where the non local propagators $G^{\alpha \beta \gamma \delta}\left(i, j ; t_{1}, t_{2}, t_{3}, t_{4}\right)$ represent the corrections at order $O(1 / N)$ to the MF correlations. By an appropriated base change, from the unidimensional spin variables $s_{i}$ to the eigenvectors $\eta^{\alpha}$ of the $J_{i j}$ matrix, we will manage to decouple the interaction. 
We define $\eta^{\alpha}$ :

$$
\sum_{j} J_{i j} \eta_{j}^{\alpha}=\lambda^{\alpha} \eta_{j}^{\alpha}
$$

where $\alpha=1 \ldots \ldots N$ and $\lambda^{\alpha}$ is the $\alpha$-th eigenvalue. The properties of the eigenvalues and the eigenvectors of the random matrix $J_{i j}$ are the following (see [2]):

the shape of the eigenvalues density $\sigma(\lambda)$, for symmetric matrices with random and statistically independent elements, such as $J_{i j}$, in the limit $N \rightarrow \infty(N$ dimension of the matrix), is:

$$
\sigma(\lambda)= \begin{cases}\frac{1}{2 \pi}\left(4-\lambda^{2}\right)^{1 / 2} & |\lambda|<2 \\ 0 & |\lambda|>2\end{cases}
$$

the eigenvectors are statistical variables which have the components independent and following a gaussian distribution defined by the moments:

$$
\begin{gathered}
\overline{\eta_{i}^{\alpha}}=0, \\
\overline{\left(\eta_{i}^{\alpha}\right)^{2 K}}=\frac{(2 K-1) ! !}{N^{K}}
\end{gathered}
$$

they are found to be orthogonal and we can choose them to be normalized:

$$
\begin{aligned}
\sum_{i=1}^{N} \eta_{i}^{\alpha} \eta_{i}^{\beta} & =\delta_{\alpha \beta}, \\
\sum_{\alpha=1}^{N}\left(\eta_{i}^{\alpha}\right) \eta_{j}^{\alpha} & =\delta_{i j}
\end{aligned}
$$

finally, the eigenvectors are uncorrelated among themselves (apart from the orthogonality constraint) and they are not correlated to the eigenvalues.

If we write $s_{i}=\sum_{\alpha} Y^{\alpha} \eta_{i}^{\alpha}$ in the base of the eigenvectors we obtain:

$$
\beta \mathcal{H}_{Y}=\frac{1}{2} \sum_{\alpha}\left(-\beta \lambda^{\alpha}+r_{0}\right)\left(Y^{\alpha}\right)^{2}+\frac{g}{4 !} \sum_{\alpha \beta \gamma \delta} Y^{\alpha} Y^{\beta} Y^{\gamma} Y^{\delta} \sum_{i} \eta_{i}^{\alpha} \eta_{i}^{\beta} \eta_{i}^{\gamma} \eta_{i}^{\delta}
$$

We can evaluate the Green functions for the component $Y^{\alpha}$ and, by using (8) and (9)-(12), go back to the correlation functions of $s_{i}$ field. 
We can start evaluating the correlation functions in the static theory. In the time-independent limit for the Hamiltonian (13) we may consider the nonlinear interaction as a perturbation and make a series expansion in the coupling constant $g$. We can show that only the diagrams considered in the Hartree-Fock approximation give relevant contributions to the free theory, in the thermodynamic limit. This is due to the relations (9)-(12) satisfied by the eigenvector $\eta_{i}$. In fact, in the MF limit the relevant contribution to the interaction term in the (13) is $\sum_{i}\left(\eta^{\alpha}\right)^{2}\left(\eta^{\beta}\right)^{2} \propto 1 / N$, and in this way one can see easily that the eigenvector index ( $\alpha$ for $Y^{\alpha}$ ) plays the same role of the component index in the theories of vector fields, where the Hartree-Fock approximation has been demonstrated valid when the number of the components goes to infinity. We can thus resum all the diagrams at the next orders in $g$ and find a renormalization of the mass term (the coefficient of the quadratic term in (13)) that for $T \rightarrow T_{c}^{+}$is

$$
m^{2} \propto\left(\frac{T-T_{c}}{T_{c}}\right)^{2}=\left(\frac{\tau}{T_{c}}\right)^{2}
$$

and a renormalization of the coupling constant $g$, that in the same region is

$$
g_{r}=\left(2 m^{2}\right)^{\frac{1}{2}}
$$

The static susceptibility shows a divergent behaviour for $T \rightarrow T_{c}^{+}$with the critical exponent $\gamma=1$, in agreement with the results obtained with the replica method 11

$$
\begin{aligned}
\chi_{S . G .}=\frac{1}{N} \sum_{i k}\left\langle s_{i} s_{k}\right\rangle_{\sigma(\lambda)}^{2} & =\int_{0}^{4} \frac{d \lambda \sqrt{\left(4 \lambda-\lambda^{2}\right)}}{2 \pi\left(\beta \lambda+m^{2}\right)^{2}} \\
& =\frac{2}{\left(2 m^{2}\right)^{1 / 2}} \propto \frac{1}{\tau} .
\end{aligned}
$$

The generic 4-point function

$$
\left.\left[\left\langle s_{i} s_{k} s_{i} s_{k}\right\rangle_{\xi}\right]_{J}=\left[\left\langle\left(s_{i}\right)^{2}\right\rangle_{\xi}\left\langle\left(s_{k}\right)^{2}\right\rangle_{\xi}\right]_{J}+2\left[\left(\left\langle s_{i} s_{k}\right\rangle_{\xi}\right)^{2}\right)\right]_{J}+\left[\left\langle s_{i} s_{k} s_{i} s_{k}\right\rangle_{\xi \text { conn }}\right]_{J}
$$


is $O(1 / N)$ order and is regular in the critical region. In fact, for $T \rightarrow T_{c}$ the first term is regular and the divergence of the second term

$$
2 \times\left(\frac{2}{\left(2 m^{2}\right)^{1 / 2}}\right),
$$

is compensated by that of the last one

$$
-\left(\left(2 m^{2}\right)^{1 / 2}\right) \times\left(\frac{2}{\left(2 m^{2}\right)^{1 / 2}}\right) \times\left(\frac{2}{\left(2 m^{2}\right)^{1 / 2}}\right)=-\frac{4}{\left(2 m^{2}\right)^{1 / 2}} .
$$

Also in the replica approach one can verify that the 4-point function (17) is not singular for $T \rightarrow T_{c}$.

In the dynamic case we have to solve the Langevin equation for the timedependent component $Y^{n}(t)$ (we put $\Gamma_{0}=1$ ):

$$
\dot{Y}^{n}=\left(\beta \lambda^{n}-r_{0}\right) Y^{n}-g \sum_{\alpha, \beta, \gamma} Y^{\alpha} Y^{\beta} Y^{\gamma} \sum_{i} \eta_{i}^{\alpha} \eta_{i}^{\beta} \eta_{i}^{\gamma} \eta_{i}^{n}+\xi^{n}
$$

If we define $G^{n}\left(t-t^{\prime}\right)=1 / 2\left\langle Y^{n}(t) \xi^{n}\left(t^{\prime}\right)\right\rangle$ and $C^{n}\left(t-t^{\prime}\right)=\left\langle Y^{n}(t) Y^{n}\left(t^{\prime}\right)\right\rangle$ we find the formal solution

$$
\begin{aligned}
& Y^{n}(t)=\int_{0}^{t} G^{n}\left(t-t^{\prime}\right) \xi^{n}\left(t^{\prime}\right) d t^{\prime}+ \\
& -g \int_{0}^{t} d t^{\prime} G^{n}\left(t-t^{\prime}\right) \sum_{\alpha, \beta, \gamma} Y^{\alpha}\left(t^{\prime}\right) Y^{\beta}\left(t^{\prime}\right) Y^{\gamma}\left(t^{\prime}\right) \sum_{i} \eta_{i}^{\alpha} \eta_{i}^{\beta} \eta_{i}^{\gamma} \eta_{i}^{n} .
\end{aligned}
$$

This is a self-consistent equation, which can be solved with an iterative procedure. At finite order in $g, Y^{n}$ is a polynomial in the variables $\xi^{n}(t)$. We can obtain the dynamic physical quantities averaging $G^{n}$ and $C^{n}$ over the distribution of the eigenvalues (8) and of the eigenvectors defined by (9)-(12). We can show that, as in the static case, only the diagrams of the Hartree-fock type are relevant in the correction to the Green functions. The correction terms do not change the dynamic behaviour of the 2-point functions for $T=T_{c}$, because the time dependent part of the self-energy is regular at $T=T_{c}$ [四. After some 
algebraic calculation we find only a mass renormalization effect for $C(\omega)$ and $G(\omega)$

$$
\begin{aligned}
G_{r}(\omega) & =\int \frac{d \lambda \sigma(\lambda)}{\left(-\beta \lambda+m^{2}-i \omega\right)} \\
& =2+\Delta T_{c}+\tau-2 \sqrt{2\left(m^{2}-i \omega\right)} \\
C_{r}(\omega) & =\int \frac{d \lambda \sigma(\lambda)}{\left(\left(-\beta \lambda+m^{2}\right)^{2}-i \omega^{2}\right)} \\
& =\frac{2 \cdot 4}{\sqrt{2\left(m^{2}-i \omega\right)}+\sqrt{2\left(m^{2}+i \omega\right)}} .
\end{aligned}
$$

where $m^{2}$ is the renormalized static parameter (14), $\Delta T_{c}=T_{c}-T_{c}^{0}$ is the difference from the renormalized and the bare critical temperature $\left(T_{c}^{0}=2\right)$, while as usually $\tau=T-T_{c}$. The relations (22) and (23) are in agreement with the critical behaviour indicated in [4] by Sompolinsky and Zippelius. The susceptibility, according to the definition (7), results

$$
\chi_{S . G .}\left(\omega_{1}, \omega_{2}\right)=\int \frac{d \lambda \sigma(\lambda)}{\left(-\beta \lambda+m^{2}-i \omega_{1}\right)\left(-\beta \lambda+m^{2}-i \omega_{2}\right)} .
$$

It is clear that $\chi_{S G}\left(\omega_{1}, \omega_{2}\right)$ has a finite limit for $\omega \rightarrow 0$ when $T>T_{c}$, while at $T=T_{c}$ it shows a critical behaviour such as

$$
\chi \propto \frac{1}{\omega^{1 / 2}} .
$$

For the generic 4-point function the connected terms occur. For example, for $\left\langle\xi_{i}\left(t_{1}\right) s_{i}\left(t_{2}\right) s_{k}\left(t_{3}\right) \xi_{k}\left(t_{4}\right)\right\rangle$, we have the diagram of Fig.[1], where, as usual, a line with an arrow represents a response function(the time order follows the arrow) and one with a cross a correlation function. The diagrams that we have to sum in order to evaluate the renormalized coupling constant are drawn in Fig. [2] and in the low frequency limit we have:

$$
g_{r}=\frac{1}{2} \sqrt{2} \sqrt{2\left(2 m^{2}-i \omega\right)}
$$


Therefore the total contribution to $\left\langle\xi_{i} s_{i} s_{k} \xi_{k}\right\rangle$ function, represented in Fig.[3], is the sum of the following terms:

1)

$$
\begin{gathered}
-\frac{1}{2} \sqrt{2}\left(\sqrt{2\left(2 m^{2}-i \bar{\omega}\right.}\right) \frac{4}{\sqrt{2\left(m^{2}-i \omega_{1}\right)}+\sqrt{2\left(m^{2}+i \omega_{2}\right)}} \times \\
\frac{1}{\sqrt{2\left(m^{2}+i \omega_{3}\right)}+\sqrt{2\left(m^{2}+i \omega_{4}\right)}} \frac{1}{\sqrt{2\left(m^{2}-i \omega_{3}\right)}+\sqrt{2\left(m^{2}+i \omega_{4}\right)}} \times \\
\frac{16}{\sqrt{2\left(m^{2}-i \omega_{3}\right)}+\sqrt{2\left(m^{2}+i \omega_{3}\right)}},
\end{gathered}
$$

2)

$$
\begin{gathered}
-\frac{1}{2} \sqrt{2}\left(\sqrt{2\left(2 m^{2}+i \bar{\omega}\right.}\right) \frac{4}{\sqrt{2\left(m^{2}-i \omega_{3}\right)}+\sqrt{2\left(m^{2}+i \omega_{4}\right)}} \times \\
\frac{1}{\sqrt{2\left(m^{2}+i \omega_{1}\right)}+\sqrt{2\left(m^{2}+i \omega_{2}\right)}} \frac{1}{\sqrt{2\left(m^{2}-i \omega_{1}\right)}+\sqrt{2\left(m^{2}+i \omega_{2}\right)}} \times \\
\frac{16}{\sqrt{2\left(m^{2}-i \omega_{1}\right)}+\sqrt{2\left(m^{2}+i \omega_{1}\right)}},
\end{gathered}
$$

3)

$$
\begin{gathered}
\frac{1}{2}\left(\frac{1}{2} \sqrt{2} \sqrt{2\left(2 m^{2}-i \bar{\omega}\right)}\right)\left(\frac{1}{2} \sqrt{2} \sqrt{2\left(2 m^{2}+i \bar{\omega}\right)}\right) \times \\
\frac{2 i \sqrt{2}}{\bar{\omega}}\left(\frac{1}{\sqrt{2\left(m^{2}-i \bar{\omega}\right)}}-\frac{1}{\sqrt{2\left(m^{2}+i \bar{\omega}\right)}}\right) \frac{4}{\sqrt{2\left(m^{2}-i \omega_{1}\right)}+\sqrt{2\left(m^{2}+i \omega_{2}\right)}} \times \\
\frac{4}{\sqrt{2\left(m^{2}-i \omega_{3}\right)}+\sqrt{2\left(m^{2}+i \omega_{4}\right)}} .
\end{gathered}
$$

In the same way we can evaluate all the 4-point functions. The static limit derived from the dynamic functions coincides, order by order in $g$, with the static results. This is true also for the renormalized functions.

It is not easy to extend this formalism to the short-range model, because in that case the eigenvalue density $\sigma(\lambda)$ is not given by the simple expression (8). Although the results of this section have been useful to us for understanding some properties of the correlation functions in the critical region, to go beyond the MF approximation we have to leave this approach. In the next section we 
will use the functional integral formalism for a dynamic SG model in finite dimensions. The results obtained in this section will be used as comparative terms for the long-range limit of the short-range correlation functions.

\section{Fluctuations in short range spin glasses. Functional integral formulation.}

To solve a theory with quenched parameters we can use the functional integral formulation for dynamics, introduced by De Dominicis [13]. So we consider an auxiliary field $\hat{s}_{i}(t)$, 12 , and we define a two-component vector field

$\phi_{i}^{\alpha}=\left(i \hat{s}_{i}, s_{i}\right)$. One can show [4] that in this formalism the factor $i \hat{s}_{i}(t)$ in a correlation function acts like $\frac{\partial}{\partial \beta h_{i}}$, where $h_{i}$ is the external field:

$$
\left\langle s_{i}(t) i \hat{s}_{i}\left(t^{\prime}\right)\right\rangle_{L(s, \hat{s})}=\frac{\partial\left\langle s_{i}(t)\right\rangle_{L(s, \hat{s})}}{\partial \beta h_{i}\left(t^{\prime}\right)}=G\left(t-t^{\prime}\right)
$$

So $i \hat{s}_{i}(t)$ replaces the noise $\xi_{i}(t)$ to generate the response functions.

After averaging over $J_{i j}$ (in this case we can avoid the replica trick because the functional $\mathcal{Z}$ is normalized), a 4 -spin interaction is generated. It is convenient to decouple this interaction by a gaussian transformation [4]. Then the theory can be defined with a generating functional in the $Q_{i}^{\alpha \beta}\left(t, t^{\prime}\right)$ variables:

$$
\begin{aligned}
\mathcal{Z}=\int \prod_{\alpha, \beta=1,2}\left[D Q_{i}^{\alpha \beta}(1,2)\right] \exp (- & d 1 \mathrm{~d} 2 \sum_{i, j} \tilde{K}_{i j}^{-1} Q_{i}^{\alpha \beta}(1,2) A^{\alpha \beta \gamma \delta} Q_{j}^{\gamma \delta}(1,2) \\
& +\ln \int[d s][d \hat{s}] \exp \left(L_{1}\left(s, \hat{s}, Q_{i}^{\alpha \beta}\right)\right)
\end{aligned}
$$

where $\tilde{K}_{i j}^{-1}=\frac{z}{\beta^{2}} K_{i j}^{-1}\left(K_{i j}=1\right.$ if $i, j$ are nearest neighbors and zero otherwise) and $A^{\alpha \beta \gamma \delta}$ is such that $A^{1122}=A^{2211}=A^{2112}=A^{1221}=1$ and 0 otherwise. $L_{1}$ is:

$$
L_{1}=L_{0}+\int d 1 d 2 Q_{i}^{\alpha \beta}(12) \psi_{i}^{\alpha}(1) \psi_{i}^{\beta}(2)
$$


where the field $\psi_{i}^{\alpha}$ is defined:

$$
\psi_{i}^{\alpha}=\left(\begin{array}{cc}
0 & 1 \\
1 & 0
\end{array}\right) \phi_{i}^{\alpha}=\left(s_{i}, i \hat{s}_{i}\right)
$$

and $L_{0}$ is the local part of the theory:

$$
L_{0}=\int d 1 \sum_{i}\left[i \hat{s}_{i}(1)\left(-\Gamma_{0}^{-1} \partial_{1} s_{i}(1)-r_{0} s_{i}(1)-\frac{1}{3 !} g s_{1}^{3}(1)+\Gamma_{0}^{-1} i \hat{s}_{i}(1)\right)\right] \text {. }
$$

The value of the Jacobi determinant $J$, associated to the integral formulation of the $\delta$ function [13] depends on the discretization chosen to regularize the Langevin equation. With the following regularization:

$$
s(t+\epsilon)-s(t)+\epsilon\left(-\frac{\partial(\beta \mathcal{H})}{\partial s_{i}(t)}\right)=D_{i}^{\epsilon}(t)
$$

where $D_{i}^{\epsilon}(t)=\int_{t}^{t+\epsilon} \xi_{i}\left(t^{\prime}\right) d t^{\prime}$, one has $J=1$ (and it has been omitted in all the previous formula).

In the previous expressions and in the followings of this section we shall write 1 for $t_{1}, 2$ for $t_{2}$ and so on.

The solution of the MF theory is well known [4], and it is consistent with the results obtained in the previous section. Let us consider the fluctuations around the saddle point value $Q_{i}^{\alpha \beta}(1,2)=\overline{Q_{i}^{\alpha \beta}}(1,2)+\delta Q_{i}^{\alpha \beta}(1,2)$. As a result the model can be formulated in terms of the dynamic fluctuation field $\delta Q_{i}^{\alpha \beta}(1,2)$ :

$$
\mathcal{Z}=\int \prod_{\alpha, \beta=1,2} D\left\{\delta Q_{i}^{\alpha \beta}\right\} \exp \left(L\left(s, \hat{s}, \overline{Q_{i}^{\alpha \beta}}+\delta Q_{i}^{\alpha \beta}\right)\right)
$$

where $L$ contains the quadratic and the cubic term of the series expansion around the MF value: 


$$
\begin{aligned}
L & =-\sum_{1,2} \sum_{i, j} \tilde{K}_{i, j}^{-1} \delta Q_{i}^{\alpha \beta}(1,2) A^{\alpha \beta \gamma \delta} \delta Q_{j}^{\gamma \delta}(1,2)+ \\
& +\frac{1}{2} \sum_{1,2,3,4} \sum_{i} \delta Q_{i}^{\alpha \beta}(1,2) C^{\alpha \beta \gamma \delta}(1,2,3,4) \delta Q_{i}^{\gamma \delta}(3,4)+ \\
& +\frac{1}{3 !} \sum_{1,2,3,4,5,6} \sum_{i} C^{\alpha \beta \gamma \delta \mu \nu}(1,2,3,4,5,6) \delta Q_{i}^{\alpha \beta}(1,2) \delta Q_{i}^{\gamma \delta}(3,4) \delta Q_{i}^{\mu \nu}(5,6) .
\end{aligned}
$$

The higher order terms can be neglected because we are interested in the behaviour of the Green functions near the critical temperature in the paramagnetic phase. The $C^{\alpha \beta \gamma \delta}(1,2,3,4)$ and $C^{\alpha \beta \gamma \delta \mu \nu}(1,2,3,4,5,6)$ vertices are the 4-spin and 6-spin correlation functions of the one site MF theory described by the partition function

$$
\mathcal{Z}_{\boldsymbol{I}}=\int\left[d s_{i}\right]\left[d \hat{s}_{i}\right] \exp \left[L_{0}+\int d 1 d 2 \sum_{i} \overline{Q_{i}^{\alpha \beta}}(1,2) \psi_{i}^{\alpha}(1) \psi_{i}^{\beta}(2)\right],
$$

connected with respect to pairs. For example for $C^{\alpha \beta \gamma \delta}(1,2,3,4)$ we have:

$C^{\alpha \beta \gamma \delta}(1,2,3,4)=\left\langle\psi_{i}^{\alpha}(1) \psi_{i}^{\beta}(2) \psi_{i}^{\gamma}(3) \psi_{i}^{\delta}(4)\right\rangle_{M F}-\left\langle\psi_{i}^{\alpha}(1) \psi_{i}^{\beta}(2)\right\rangle_{M F}\left\langle\psi_{i}^{\gamma}(3) \psi_{i}^{\delta}(4)\right\rangle_{M F}$.

The form of the functions $C^{\alpha \beta \gamma \delta}$ and $C^{\alpha \beta \gamma \delta \mu \nu}$ is crucial in the following. We want to study the universal behaviour of the system near the critical fixed point, so we are interesting in the singular part of these functions for $T=T_{c}$, and for $\omega \rightarrow 0$. Thus we consider $T=T_{c}$ from the beginning in the MF theory described by the functional (38) for the soft-spin model. Because of we are not able to compute the 4-point and 6-point functions analytically in a closed simple form, a perturbative approach will be used. We perform an expansion in the quartic vertex $\left[\frac{1}{3 !} g\left(s_{i}\right)^{3} i \hat{s}_{i}\right]$ using the MF expressions (22) and (23) for the propagators $\left[\left\langle s_{i}(t) i \hat{s}_{i}\left(t^{\prime}\right)\right\rangle\right]_{J}$ and $\left[\left\langle s_{i}(t) s_{i}\left(t^{\prime}\right)\right\rangle\right]_{J}$ respectively $\left(\left[\left\langle i \hat{s}_{i}(t) i \hat{s}_{i}\left(t^{\prime}\right)\right\rangle\right]_{J} \equiv 0\right)$. 
We can demonstrate that the critical behaviour of these functions is determined only by the zero loop contributions. In fact the loops that we can form with the quartic vertex of $L_{0}$, in the $\omega$ space, are:

The first is infrared converging and the latter is only logarithmic infrared diverging. In the evaluation of any renormalized correlation function, these diverging loops occur always multiplied by correlation functions which are less singular (for $T \rightarrow T_{c}$ ) than the tree level ones. For example, for the function $C^{1221}\left(\omega_{1}, \omega_{2}, \omega_{3}, \omega_{4}\right)$ we obtain:

$$
\begin{gathered}
C^{1221}\left(\omega_{1}, \omega_{2}, \omega_{3}, \omega_{4}\right)=G\left(\omega_{4}\right) G\left(\omega_{1}\right) \delta\left(\omega_{4}+\omega_{2}\right) \delta\left(\omega_{1}+\omega_{3}\right)+ \\
g \cdot G\left(-\omega_{2}\right) G\left(-\omega_{3}\right)\left[C\left(\omega_{1}\right) G\left(\omega_{4}\right)+C\left(\omega_{4}\right) G\left(\omega_{1}\right)\right] \delta\left(\omega_{1}+\omega_{2}+\omega_{3}+\omega_{4}\right)+ \\
g^{2} \cdot G\left(\omega_{1}\right) G\left(\omega_{2}\right) G\left(\omega_{3}\right) G\left(\omega_{4}\right) \cdot \log (\bar{\omega}) \delta\left(\omega_{1}+\omega_{2}+\omega_{3}+\omega_{4}\right) .
\end{gathered}
$$

From the expression (22) and (23) for the response and correlation functions, we can see that the 1-loop contribution to the connected part of $C^{1221}$ is negligible (it is of order $\log (\omega)$ in the limit $\omega \rightarrow 0$ ) with respect to the zero loop one (that is of order $1 /(\omega)^{1 / 2}$ in the same limit). In the same way, we find for $C^{2221}$ that only the connected part is different from zero and is

$$
g G\left(-\omega_{2}\right) G\left(-\omega_{3}\right) G\left(-\omega_{1}\right) G\left(\omega_{4}\right) \delta\left(\omega_{1}+\omega_{2}+\omega_{3}+\omega_{4}\right) .
$$

It is reasonable to assume that this phenomenon, which we have seen in a perturbative expansion in $g$, holds beyond the perturbative theory, so, in order to study the critical behaviour of the system we can neglect the loop contributions. In [7] these correlation functions were not considered at the critical point and a factorized functional expression was proposed for them in the hard-spin 
limit. The functional form that we obtain is obviously different, but we have not yet verified if we obtain also a different value for the universal physical quantities (i.e. critical exponents).

The correlations of the $\phi_{i}^{\alpha}$ fields, which we are interested in, are related to those $\delta Q_{i}^{\alpha \beta}$ by the relations:

$$
\begin{gathered}
{\left[\left\langle\phi_{i}^{\alpha}(1) \phi_{i}^{\beta}(2)\right\rangle_{\xi}\right]_{J}=2 \sum_{j}\left(\tilde{K}^{-1}\right)_{i j}\left(\overline{Q_{j}^{\alpha \beta}}(1,2)+\left\langle\delta Q_{j}^{\alpha \beta}(1,2)\right\rangle_{L\left(Q_{\alpha \beta}\right)}\right),} \\
{\left[\left\langle\phi_{i}^{\alpha}(1) \phi_{i}^{\beta}(2) \phi_{k}^{\gamma}(3) \phi_{k}^{\delta}(4)\right\rangle_{\xi}\right]_{J}=4 \sum_{j}\left(\tilde{K}^{-1}\right)_{i j} \sum_{l}\left(\tilde{K}^{-1}\right)_{k l}} \\
\left\langle\left(\overline{Q_{j}^{\alpha \beta}}(1,2)+\delta Q_{j}^{\alpha \beta}(1,2)\right)\left(\overline{Q_{l}^{\gamma \delta}}(3,4)+\delta Q_{l}^{\gamma \delta}(3,4)\right)\right\rangle_{L\left(Q_{\alpha \beta}\right)}+ \\
-2\left(\tilde{K}^{-1}\right)_{i k} A^{\alpha \beta \gamma \delta} \delta(1-3) \delta(2-4)
\end{gathered}
$$

In the next section, therefore, we will to evaluate the correlation functions of the fields $\delta Q_{i}^{\alpha \beta}(1,2)$.

\subsection{The Propagators}

Let us consider the expression (37) with the vanishing cubic interaction. The generic propagator

$$
\begin{aligned}
& G^{\alpha \beta \gamma \delta}(i-j ; 1,2,3,4)=\left[\left\langle\phi_{i}^{\alpha}(1) \phi_{i}^{\beta}(2) \phi_{j}^{\gamma}(3) \phi_{j}^{\delta}(4)\right\rangle_{\xi}\right]_{J}-\left[\left\langle\phi_{i}^{\alpha}(1) \phi_{i}^{\beta}(2)\right\rangle_{\xi}\right]_{J}\left[\left\langle\phi_{j}^{\gamma}(3) \phi_{j}^{\delta}(4)\right\rangle_{\xi}\right]_{J} \\
= & 4 \sum_{l}\left(\tilde{K}^{-1}\right)_{i l} \sum_{k}\left(\tilde{K}^{-1}\right)_{j k}\left\langle\delta Q_{l}^{\alpha \beta}(1,2) \delta Q_{k}^{\gamma \delta}(3,4)\right\rangle_{L\left(Q_{\alpha \beta}\right)}-2\left(\tilde{K}^{-1}\right)_{i j} A^{\alpha \beta \gamma \delta} \delta(1-3) \delta(2-4),
\end{aligned}
$$

is calculated in free theory and will be used to evaluate the corrections of terms in the loop expansion. In the free theory $G^{\alpha \beta \gamma \delta}(i-j ; 1,2,3,4)$ is the solution to the following integral system:

$$
\sum_{3,4}\left(-2 \tilde{K}^{-1}(k) A^{\alpha \beta \gamma \delta} \delta(1-3) \delta(2-4)+C^{\alpha \beta \gamma \delta}(1,2,3,4)\right) \times
$$




$$
\begin{aligned}
\left(\frac{1}{4} G^{\gamma \delta \mu \nu}(k ; 3,4,5,6)+\right. & \left.\frac{1}{2} \tilde{K}^{-1}(k) A^{\gamma \delta \mu \nu} \delta(3-5) \delta(4-6)\right)= \\
& =-\delta_{\alpha, \mu} \delta_{\beta, \nu} \tilde{K}^{-2}(k) \delta(1-5) \delta(2-6)
\end{aligned}
$$

For each value of $\mu$ and $\nu$ (45) is an integral system of four coupled equations in the $G^{11 \mu \nu}, G^{21 \mu \nu}, G^{12 \mu \nu}, G^{22 \mu \nu}$ variables. Obviously we consider $G^{\alpha \beta \mu \nu}\left(i, j ; t_{1}, t_{2}, t_{3}, t_{4}\right)$ invariant under permutation of the index $\alpha, \beta, \mu, \nu$ and of the relatives times $t_{1}, t_{2}, t_{3}, t_{4}$. First we solve the system for $\mu, \nu=1,2$. We are not able to solve the system (45) exactly, so we use a recursive procedure by considering $G^{\alpha \beta \gamma \delta}$ as a perturbative series in $g$. For $g=0$ the coefficients $C^{\alpha \beta 12}(1,2,3,4)$ are factorized in the time-difference and the integral kernel show a complete separation of the internal time. This is the limit in which the propagators have been computed in [5]. In Fourier space we obtain:

$$
\begin{gathered}
G_{0}^{1121}\left(k ; \omega_{1}, \omega_{2}, \omega_{3}, \omega_{4}\right)=0, \\
G_{0}^{2121}\left(k ; \omega_{1}, \omega_{2}, \omega_{3}, \omega_{4}\right)=\frac{\tilde{K}^{-1}(k) G\left(\omega_{1}\right) G\left(-\omega_{2}\right) \delta\left(\omega_{1}+\omega_{4}\right) \delta\left(\omega_{2}+\omega_{3}\right)}{\tilde{K}^{-1}(k)-G\left(\omega_{1}\right) G\left(-\omega_{2}\right)}, \\
G_{0}^{2221}\left(k ; \omega_{1}, \omega_{2}, \omega_{3}, \omega_{4}\right)=\frac{1}{\tilde{K}^{-1}(k)-G\left(\omega_{1}\right) G\left(\omega_{2}\right)}\left[C\left(\omega_{3}\right) G\left(-\omega_{4}\right) \times\right. \\
\left.\frac{\tilde{K}^{-1} G\left(\omega_{3}\right) G\left(-\omega_{4}\right)\left[\delta\left(\omega_{1}+\omega_{3}\right) \delta\left(\omega_{2}+\omega_{4}\right)+\delta\left(\omega_{1}+\omega_{4}\right) \delta\left(\omega_{2}+\omega_{3}\right)\right]}{\tilde{K}^{-1}(k)-G\left(\omega_{3}\right) G\left(-\omega_{4}\right)}\right] .
\end{gathered}
$$

Also at the zero order of the perturbation series in $g$ we consider the mass term $\left(m^{2}\right)$ renormalized by the interaction.

At low frequency and for $T \rightarrow T_{c}$ we have $\tilde{K}^{-1}(k)=\frac{1}{\beta^{2}} K^{-1}(k)=\left(4+4 \Delta T_{c}+4 \tau\right)\left(1+c k^{2}\right), G(\omega)$ given by the (22) and $C(\omega)$ by the (23) and the expression (47) and (48) become:

$$
G_{0}^{2121}\left(k ; \omega_{1}, \omega_{2}, \omega_{3}, \omega_{4}\right)=\frac{4 \delta\left(\omega_{1}+\omega_{4}\right) \delta\left(\omega_{2}+\omega_{3}\right)}{c k^{2}+\sqrt{2\left(m^{2}-i \omega_{1} / \Gamma_{0}\right)}+\sqrt{2\left(m^{2}+i \omega_{2} / \Gamma_{0}\right)}},
$$




$$
\begin{gathered}
G_{0}^{2221}\left(k ; \omega_{1}, \omega_{2}, \omega_{3}, \omega_{4}\right)=\frac{\delta\left(\omega_{1}+\omega_{3}\right) \delta\left(\omega_{2}+\omega_{4}\right)+\delta\left(\omega_{1}+\omega_{3}\right) \delta\left(\omega_{2}+\omega_{4}\right)}{4\left(c k^{2}+\sqrt{2\left(m^{2}-i \omega_{1} / \Gamma_{0}\right)}+\sqrt{2\left(m^{2}-i \omega_{2} / \Gamma_{0}\right)}\right)} \times \\
\frac{4}{\left(c k^{2}+\sqrt{2\left(m^{2}-i \omega_{3} / \Gamma_{0}\right)}+\sqrt{2\left(m^{2}+i \omega_{4} / \Gamma_{0}\right)}\right)} \times \\
\frac{4 \cdot 2 \cdot 2}{\left(\sqrt{2\left(m^{2}-i \omega_{3} / \Gamma_{0}\right)}+\sqrt{2\left(m^{2}+i \omega_{3} / \Gamma_{0}\right)}\right)}
\end{gathered}
$$

In the same way, if we calculate the system for $\mu, \nu=2,2$ we obtain the propagator $G^{2222}$ that for $g=0$ results:

$$
\begin{gathered}
G_{0}^{2222}\left(k ; \omega_{1}, \omega_{2}, \omega_{3}, \omega_{4}\right)=\frac{\delta\left(\omega_{1}+\omega_{3}\right) \delta\left(\omega_{2}+\omega_{4}\right)}{4\left(c k^{2}+\sqrt{2\left(m^{2}-i \omega_{1} / \Gamma_{0}\right)}+\sqrt{2\left(m^{2}-i \omega_{2} / \Gamma_{0}\right)}\right)} \times \\
{\left[\frac{2}{\left(c k^{2}+\sqrt{2\left(m^{2}+i \omega_{3} / \Gamma_{0}\right)}+\sqrt{2\left(m^{2}-i \omega_{4} / \Gamma_{0}\right)}\right)}+\right.} \\
\left.+\frac{2}{\left(c k^{2}+\sqrt{2\left(m^{2}-i \omega_{3} / \Gamma_{0}\right)}+\sqrt{2\left(m^{2}+i \omega_{4} / \Gamma_{0}\right)}\right.}\right] \times \\
\frac{2 \cdot 4 \cdot 2}{\left(\sqrt{2\left(m^{2}-i \omega_{2} / \Gamma_{0}\right)}+\sqrt{2\left(m^{2}+i \omega_{2} / \Gamma_{0}\right)}\right)} \frac{2}{\left(\sqrt{2\left(m^{2}-i \omega_{3} / \Gamma_{0}\right)}+\sqrt{2\left(m^{2}+i \omega_{3} / \Gamma_{0}\right)}\right)} \times \\
\frac{1}{\left(c k^{2}+\sqrt{2\left(m^{2}-i \omega_{3} / \Gamma_{0}\right)}+\sqrt{2\left(m^{2}-i \omega_{4} / \Gamma_{0}\right)}\right)},
\end{gathered}
$$

plus another term of the same form proportional to $\left(\delta\left(\omega_{1}+\omega_{4}\right) \delta\left(\omega_{2}+\omega_{3}\right)\right)$.

For $\chi_{S . G .}(k, \omega)=G^{2121}\left(k ; \omega=\omega_{1}=\omega_{2}\right)$ we obtain the following scaling behaviour for $T \rightarrow T_{c}^{+}$:

$$
\chi_{S . G .}(k, \omega)=\xi^{2-\eta} f\left(k \xi, \omega \xi^{z}\right)
$$

where $\xi$ is the correlation length and $\eta$ and $z$ are the usual critical exponents that, at the MF approximation, take the value of 0 and 4 respectively.

At the first order in $g$ we consider $G^{\alpha \beta \gamma \delta}=G_{0}^{\alpha \beta \gamma \delta}+G_{1}^{\alpha \beta \gamma \delta}$. For $G_{0}^{\alpha \beta \gamma \delta}$ we use the former solution and we solve the system in the $G_{1}$ variables. We apply this procedure in an iterative way and we can show that, defining from 
a diagrammatic point of view

$$
\begin{aligned}
& G_{0}^{1221}\left(i-j ; \omega_{1}, \omega_{2}, \omega_{3}, \omega_{4}\right)=G_{0}^{1221}\left(i-j ; \omega_{1}, \omega_{2}\right)= \\
& G_{0}^{2221}\left(i-j ; \omega_{1}, \omega_{2}, \omega_{3}, \omega_{4}\right)=G_{0}^{2221}\left(i-j ; \omega_{1}, \omega_{2}\right)= \\
& G_{0}^{2222}\left(i-j ; \omega_{1}, \omega_{2}, \omega_{3}, \omega_{4}\right)=G_{0}^{2222}\left(i-j ; \omega_{1}, \omega_{2}\right)=
\end{aligned}
$$

we obtain, for the following orders in $g$, recursive expressions that can be resumed. For istance, the terms that occur for the function $G^{1221}\left(i-j ; \omega_{1}, \omega_{2}, \omega_{3}, \omega_{4}\right)$ can be represented with the diagrams in Fig. [4].

The expansion that we obtain can be seen as a perturbative series in the quadratic vertex

$$
C_{\text {conn. }}^{2221}(1,2,3,4) \delta Q_{i}^{22}(1,2) \delta Q_{i}^{21}(34)
$$

In fact, we evaluate the free propagator $G_{0}$ considering only the disconnected part of the $C^{\alpha \beta \gamma \delta}(1,2,3,4$, ), and we use a perturbative approach to take account of the connected part. The number of the topologically different vertices of form

$$
C_{\text {conn. }}^{\alpha \beta \gamma \delta}(1,2,3,4) \delta Q_{i}^{\alpha \beta}(1,2) \delta Q_{i}^{\gamma \delta}(34)
$$

are 4 (the number of the superscripts equals one or two, and $C^{2222} \equiv 0$ ). By an explicit computation, one can see that the diagrams obtained with the vertex (56) are the most diverging ones.

Indeed, for example, the contribution to the function $\left\langle\delta Q^{12}(1,2) \delta Q^{12}(3,4)\right\rangle$ at the first order in $\mathrm{g}$ from the vertex (56) is

$$
\begin{gathered}
\left\langle\delta Q^{12}(1,2) \delta Q^{12}(3,4) \delta Q^{22}(5,6) \delta Q^{21}(7,8) C_{\text {conn }}^{2221}(5,6,7,8)\right\rangle \propto \\
\left\langle\delta Q^{12}(1,2) \delta Q^{22}(5,6)\right\rangle\left\langle\delta Q^{12}(3,4) \delta Q^{21}(7,8)\right\rangle C_{\text {conn }}^{2221}(5,6,7,8) \stackrel{k, \omega \rightarrow 0}{\longrightarrow} \\
\frac{1}{\omega^{3 / 2}} \cdot \frac{1}{\omega^{1 / 2}} \cdot g \cdot 1=g \cdot\left(1 / \omega^{2}\right)
\end{gathered}
$$


as one can verify from (22) (23) and (41), while from the vertex

$$
C_{\text {conn. }}^{2121}(5,6,7,8) \delta Q^{21}(5,6) \delta Q_{i}^{21}(7,8)
$$

one obtains a weaker singularity of order:

$$
\begin{gathered}
\left\langle\delta Q^{12}(1,2) \delta Q^{12}(3,4) \delta Q^{21}(5,6) \delta Q^{21}(7,8) C_{\text {conn }}^{2121}(5,6,7,8)\right\rangle \propto \\
\left\langle\delta Q^{12}(1,2) \delta Q^{21}(5,6)\right\rangle\left\langle\delta Q^{12}(3,4) \delta Q^{21}(7,8)\right\rangle C_{\text {conn }}^{2121}(5,6,7,8) \stackrel{k, \omega \rightarrow 0}{\longrightarrow} \\
\frac{1}{\omega^{1 / 2}} \cdot \frac{1}{\omega^{1 / 2}} \cdot g \cdot \frac{1}{\omega^{1 / 2}}=g \cdot\left(1 / \omega^{3 / 2}\right) .
\end{gathered}
$$

At this order in $g$ we do not have any other contribution to $\left\langle\delta Q^{12}(1,2) \delta Q^{12}(3,4)\right\rangle$, because the corrections, that one can obtain with the vertices

$$
C_{\text {conn. }}^{2111}(5,6,7,8) \delta Q^{21}(5,6) \delta Q^{11}(7,8)
$$

and

$$
C_{\text {conn. }}^{1111}(5,6,7,8) \delta Q_{i}^{11}(5,6) \delta Q_{i}^{11}(7,8)
$$

involved the propagator $\left\langle\delta Q^{12} \delta Q_{i}^{11}\right\rangle$ that are vanishing at the zero order in $g$.

Similarly, other functions can be calculated to verify that stronger singularity all arise from the vertex (56).

Therefore, as usual in the case of expansion in a quadratic vertex, we can resum the series. Moreover we deal with an order parameter depending on two times and we have to consider, in the Fourier space, the integral over the free internal frequencies.

To evaluate the complete propagators at $T=T_{c}$, we can define the renormalized coupling constant

$$
g_{r}=g-g^{2} I(\bar{\omega}, k)+g^{3} I^{2}(\bar{\omega}, k)+\ldots \ldots . .=\frac{g}{1+g I(\bar{\omega}, k)},
$$


where $I(\bar{\omega}, k)$, correspondent to the loop

is given by the integral: 


$$
\begin{gathered}
I_{1}(\bar{\omega}, k)=\int_{-\infty}^{\infty} \frac{d \omega}{2 \pi} \frac{1}{\left(c k^{2}+\sqrt{-2 i \omega / \Gamma_{0}}+\sqrt{-2 i(\bar{\omega}-\omega) / \Gamma_{0}}\right.} \times \\
\frac{1}{\left(c k^{2}+\sqrt{2 i \omega / \Gamma_{0}}+\sqrt{-2 i(\bar{\omega}-\omega) / \Gamma_{0}}\right.} \frac{16}{\sqrt{-2 i \omega / \Gamma_{0}}+\sqrt{2 i \omega / \Gamma_{0}}} \times \\
=\frac{1}{c k^{2}+\sqrt{-2 i \bar{\omega} / \Gamma_{0}}} F_{1}\left(\frac{c k^{2}}{\left(i \bar{\omega} / \Gamma_{0}\right)^{1 / 2}}\right) .
\end{gathered}
$$

$F_{1}$ is a function of the variable $\left(\frac{c k^{2}}{(i \bar{\omega})^{1 / 2}}\right)$ that exhibits a constant limit for $\omega \rightarrow 0$ and for $k \rightarrow 0$. The coupling constant in the low frequency and small moments limit is, according to (59) and to (60),

$$
g_{r}=\left(c k^{2}+\sqrt{-2 i \bar{\omega} / \Gamma_{0}}\right) 1 / F_{1} .
$$

To evaluate the total contribution of the loop corrections we must consider the term given by the following diagram:

with renormalized vertices. The value of the loop is:

$$
\begin{gathered}
I_{2}(\bar{\omega}, k)=\int_{-\infty}^{\infty}\left[\frac{d \omega}{2 \pi} \frac{1}{c k^{2}+\sqrt{-2 i \omega / \Gamma_{0}}+\sqrt{-2 i(\bar{\omega}-\omega) / \Gamma_{0}}} \frac{1}{c k^{2}+\sqrt{2 i \omega / \Gamma_{0}}+\sqrt{2 i(\bar{\omega}-\omega) / \Gamma_{0}}} \times\right. \\
\left(\frac{1}{c k^{2}+\sqrt{-2 i \omega / \Gamma_{0}}+\sqrt{2 i(\bar{\omega}-\omega) / \Gamma_{0}}}+\frac{1}{c k^{2}+\sqrt{2 i \omega / \Gamma_{0}}+\sqrt{-2 i(\bar{\omega}-\omega) / \Gamma_{0}}}\right) \times \\
\left.\frac{8}{\sqrt{-2 i \omega / \Gamma_{0}}+\sqrt{2 i \omega / \Gamma_{0}}} \frac{8}{\sqrt{-2 i(\bar{\omega}-\omega) / \Gamma_{0}}+\sqrt{2 i(\bar{\omega}-\omega) / \Gamma_{0}}}\right] \times \\
=\frac{1}{c^{2} k^{4}+2 i \bar{\omega} / \Gamma_{0}}\left(\frac{1}{c k^{2}+\sqrt{2 i \bar{\omega} / \Gamma_{0}}}\right) F_{2}\left(\frac{c k^{2}}{\left(i \bar{\omega} / \Gamma_{0}\right)^{1 / 2}}\right)
\end{gathered}
$$

where $F_{2}(x)$, like $F_{1}(x)$, has a constant limit for $\omega \rightarrow 0$ and $k \rightarrow 0$. 
The connected part of the free propagator $G^{1221}$ at $T=T_{c}$ is computed adding up all the diagrams in Fig.[4]. We have:

$$
\begin{gathered}
G^{1221}\left(k ; \omega_{1}, \omega_{2}, \omega_{3}, \omega_{4}\right)_{c o n n}=\left[G_{0}^{1221}\left(k ; \omega_{1}, \omega_{2}\right) G_{0}^{2122}\left(k ; \omega_{3}, \omega_{4}\right) g_{r}(k ; \bar{\omega}) \frac{1}{F_{1}}+\right. \\
+G_{0}^{1222}\left(k ; \omega_{1}, \omega_{2}\right) G_{0}^{2121}\left(k ; \omega_{3}, \omega_{4}\right) g_{r}(k ;-\bar{\omega}) \frac{1}{F_{1}}+ \\
+G_{0}^{1221}\left(k ; \omega_{1}, \omega_{2}\right) G_{0}^{2121}\left(k ; \omega_{3}, \omega_{4}\right) g_{r}(k ; \bar{\omega}) g_{r}(k ;-\bar{\omega}) \frac{1}{F_{1}^{2}} \\
\left.\frac{1}{c^{2} k^{4}+2 i \bar{\omega} / \Gamma_{0}}\left(\frac{1}{c k^{2}+\sqrt{2 i \bar{\omega} / \Gamma_{0}}}\right) F_{2}\right] \delta(\omega 1+\omega 2+\omega 3+\omega 4)
\end{gathered}
$$

which corresponds to the diagrams in Fig. [5] with the constant $g_{r}$ given by (61) and $\bar{\omega}=\omega_{2}-\omega_{1}$.

\section{Conclusion}

The connected term of the propagators is zero only in the limit of two complete time separations. In all the other cases we must calculate the complete correlation function. In this way we compute $G^{\alpha \beta \gamma \delta}$ for all the values of the indices $\alpha \beta \gamma \delta$ and for all the time distances. It is easy to see that the long-range limit $(k \rightarrow 0)$ of the connected part of the expressions (63) coincides with the sum of the terms (27) (28) (29) of the third section. On the level of the Gaussian approximation, i.e. cubic interactions are neglected, the connected part of the propagators does not contribute to the susceptibility and the dynamic scaling (52) is correct.

Therefore, we have analysed the critical behaviour of the propagators of the soft-spin model in the quadratic approximation and we have put the bases for a short-range theory of SG in the renormalization group formalism. In fact the expressions that we have derived could be used to evaluate the contributions of 
the Feynmann diagrams that occur in the loop expansion when the cubic term of the Lagrangian (37) is considered non vanishing. The expression which we have obtained in the soft-spin case are quite different from those obtained by Zippelius in ref. [7]. It is certainly interesting to understand if the value of the dynamical critical exponent is affected by this difference. The computation of the loops will be crucial to clarify this point.

\section{Acknowledgments}

I am grateful to Giorgio Parisi for his support, essential for the realization of this work. I also would like to thank Enzo Marinari for useful discussion and suggestion. 


\section{References}

[1] M. Mezard, G. Parisi and M.A. Virasoro, Spin glasses theory and beyond, World Scientific, Singapore (1987).

[2] M.L. Mehta, Random matrices, Academic, New York (1967).

[3] H. Sompolinsky and A. Zippelius, Phys. Rev. Lett., 47, 359 (1981).

[4] H. Sompolinsky and A. Zippelius, Phys. Rev. B, 25, 6860 (1982).

[5] H. Sompolinsky and A. Zippelius, Phys. Rev. lett, 50, 1297 (1983).

[6] H. Sompolinsky, Phys. Rev. Lett., 47, 935 (1981).

[7] A. Zippelius, Phys. Rev. B , 29, 2717 (1984).

[8] C.C. Paulsen, S.J. Williamson, H. Maletta Heidelberg Colloquium on Glassy Dynamics Proceedings, Heildeberg (1986)

[9] A.T. Ogielski, Phys. Rev. B, 32, 7384 (1985).

[10] A.P. Young, Phys. Rev. Lett., 50, 917 (1983).

[11] E. Marinari, G. Parisi, F. Ritort J. Phys. A, 27, 2687 (1994).

[12] P.C. Martin, E.D. Siggia, and H.A. Rose, Phys. Rev. A, 8, 423 (1978).

[13] C. De Dominicis and L. Peliti, Phys. Rev. B, 18, 353 (1978).

[14] P.C. Hohenberg and B.I. Halperin, Rev. Mod. Phys., 49,435 (1977).

[15] P.C. Hohenberg, B.I. Halperin and S.-K. Ma, Phys. Rev. Lett., 29, 1548 (1972).

[16] A.B. Harris, T.C. Lubensky and J.-H. Chen, Phys. Rev. Lett., 36, 415 (1976). 
[17] J.H. Chen and T.C. Lubensky, B Phys. Rev. B, 16, 2106 (1977).

[18] J.E. Green, J. Phys. A, L43, (1985). 


\section{CAPTIONS FOR ILLUSTRATIONS}

Fig. 1: Diagrams for the correlation function $\left[\left\langle\xi_{i} s_{i} s_{k} \xi_{k}\right\rangle\right]$ in the series expansion in $g$.

Fig. 2: 1-particle irreducible diagrams that contribute to the renormalization of the coupling constant.

Fig. 3: The renormalized function $\left[\left\langle\xi_{i} s_{i} s_{k} \xi_{k}\right\rangle\right]_{\text {conn }}$.

Fig. 4: Diagrammatic representation of the propagator $G^{1221}\left(i-j ; \omega_{1}, \omega_{2}, \omega_{3}, \omega_{4}\right)$ in the series expansion in $g$.

Fig. 5: The connected part of the propagator $G^{1221}\left(i-k ; \omega_{1}, \omega_{2}, \omega_{3}, \omega_{4}\right)$. 